\title{
Painting fluid motion using convolutional neural networks: An album of fluid motion 2.0
}

\author{
Maxime Bassenne (1), Andrew Banko, and Sadaf Sobhani \\ Center for Turbulence Research, Stanford University, Stanford, California 94305, USA
}

(Received 25 January 2019; published 24 October 2019)

\begin{abstract}
This paper is associated with a poster winner of a 2018 APS/DFD Gallery of Fluid Motion Award for work presented at the DFD Gallery of Fluid Motion. The original poster is available online at the Gallery of Fluid Motion, https://doi.org/10.1103/APS.DFD.2018. GFM.P0004
\end{abstract}

DOI: 10.1103/PhysRevFluids.4.100513

In 1982, Milton Van Dyke published An Album of Fluid Motion [1], a unique collection of over 300 black-and-white photographs illustrating a diverse set of fluid phenomena. Although often categorized as a form of art, photography is arguably the most common technique used in experimental fluid mechanics research. Over the years, many photography methods, such as shadowgraphy [2], schlieren imagining [3], chronophotography [4], long exposures [5], and high-speed frame acquisition [6], have been utilized for flow visualization and as tools to study phenomena that are hidden from the human eye.

Now imagine if these visualizations served not just their scientific purpose but became the inspiration of an artistic masterpiece. What if Van Gogh, instead of looking to the night sky outside of his window at the Saint-Paul asylum, was able to look at other parts of nature for inspiration, such as a shock wave or flow instability? In this work, a convolutional neural network (CNN) is used to merge the contents of a scientific image with the style of an artistic picture, giving a glimpse into the artist's expression of classical fluid mechanics phenomena.

Convolutional neural networks are famously known for their application in image recognition [7]. Their success hinges on the ability of the network to encode the content of the image via the successive action of the network's layers, which act as filters. The output of deeper layers represents more basic, global content of the image and contains less information on the actual pixel colors [8]. For example, the placement of edges identified by an edge filter, perhaps outlining droplets in a spray, is a good representation of the image content. On the other hand, the artistic style of an image should correspond to qualities like brush stroke texture and not to the global placement of features. Remarkably, textural information is encoded by correlations between the CNN's filters and can be separated from the content information [8].

In this work, synthetic images are generated by combining the content of scientific photographs and the style of artistic paintings following the method described in [8] and implemented in an open source code [9]. Specifically, the pixels of an initially random image were updated to minimize the weighted sum of the content error between the synthetic image and photograph and

\footnotetext{
*Corresponding author: bassenne@stanford.edu
}

Published by the American Physical Society under the terms of the Creative Commons Attribution 4.0 International license. Further distribution of this work must maintain attribution to the author(s) and the published article's title, journal citation, and DOI. 

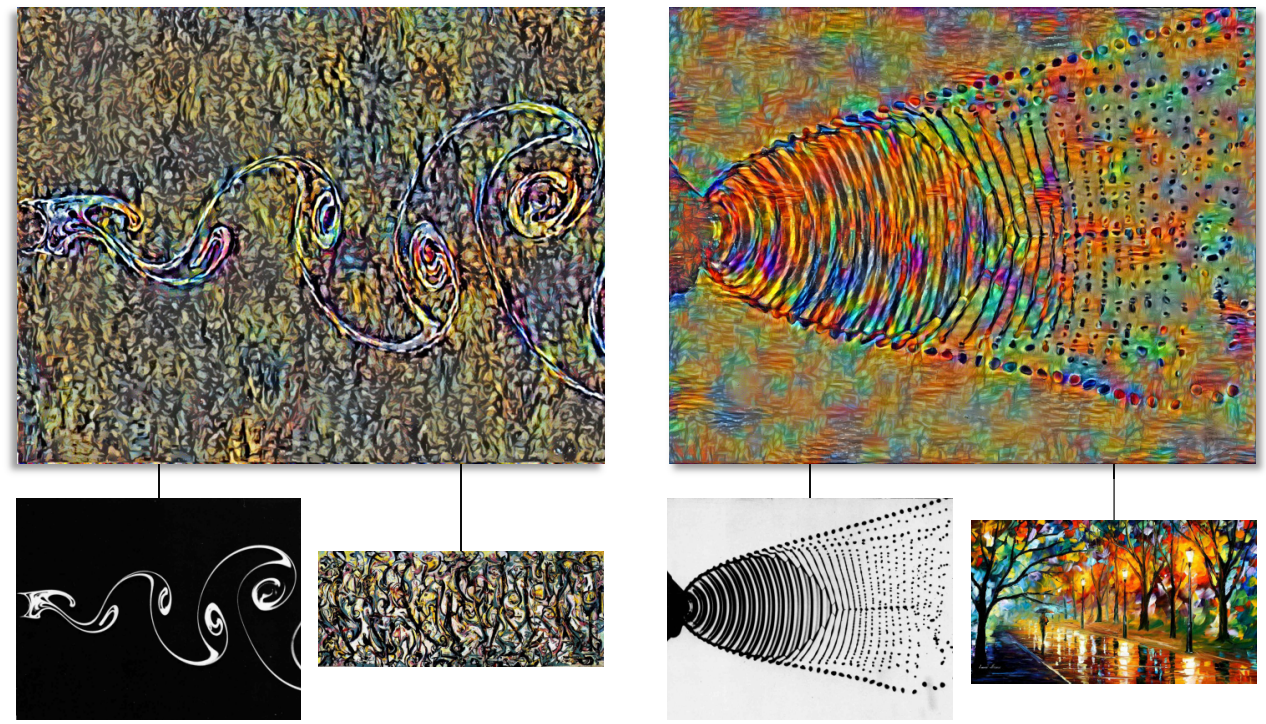

(a)

(b)

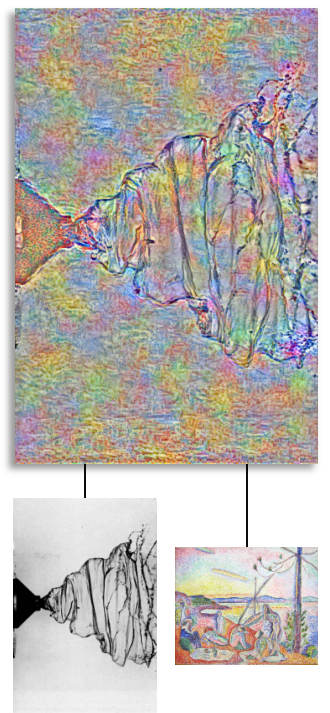

(c)

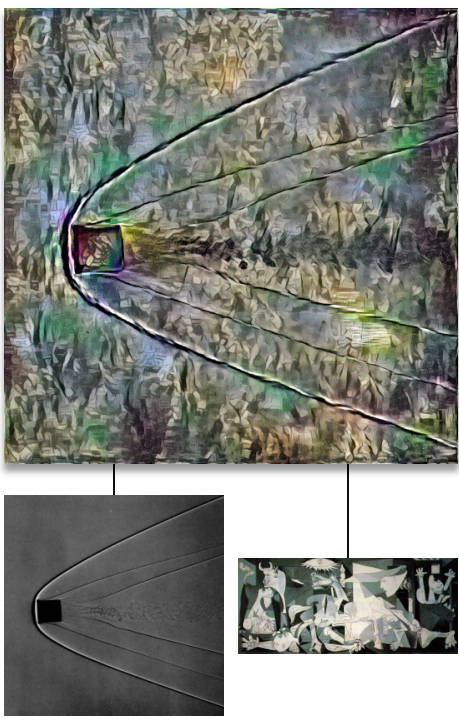

(d)

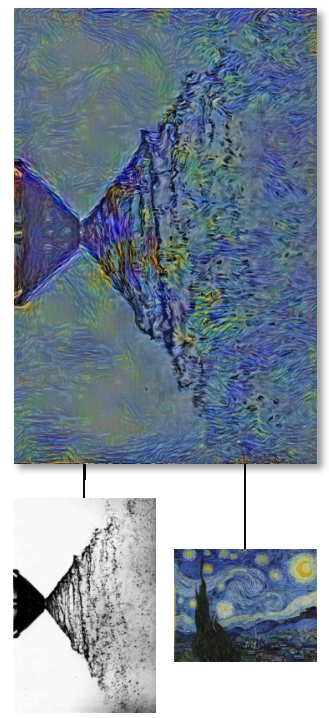

(e)

FIG. 1. Selection of paintings generated by the algorithm for five different combinations of photographs and artworks. Photographs are taken from An Album of Fluid Motion [1]: (a) 94. Kármán Vortex Street behind a Circular Cylinder at $R=140$ by S. Taneda, (b) 149. Breakup of a Liquid Sheet by N. Dombrowski, (c) and (e) 198. Atomization from a Nozzle by E. Klein, and (d) 265. Cylinder at M=3.6 in Air by A. C. Charters. Artworks: (a) Mural by Jackson Pollock, (b) Recollection of the Past by Leonid Afremov, (c) Luxe, Calme et Volupté by Henri Matisse, (d) Guernica by Pablo Picasso, and (e) The Starry Night by Vincent van Gogh. https://doi.org/10.1103/APS.DFD.2018.GFM.P0004 
the style error between the synthetic image and painting. These errors are defined as the difference between the filter outputs and filter correlations produced by feeding each image pair through the network, respectively. Figure 1 shows the synthetic images generated by the method for five different combinations of photographs and artworks. Photographs are taken from An Album of Fluid Motion [1]. With the help of artificial intelligence, these prints are transformed to reach their full artistic potential, opening new avenues for insights and inspiration.

The authors are grateful to A. Mishra, L. Jofre, K. Katan, R. Chan, A. Lozano-Durán, M. Cho, and P. Moin for useful discussions. M.B. acknowledges support from the Advanced Simulation and Computing (ASC) program of the U.S. Department of Energy's National Nuclear Security Administration via the PSAAP-II Center at Stanford (Grant No. 107908). A.B. and S.S. acknowledge the support of Bosch Corporation and the National Science Foundation Graduate Research Fellowship (Grant No. 1656518), respectively.

[1] M. D. Van Dyke, An Album of Fluid Motion (Parabolic, Stanford, CA, 1982).

[2] R. Camassa, D. M. Harris, D. Holz, R. M. McLaughlin, K. Mertens, P.-Y. Passaggia, and C. Viotti, Variable density vortex ring dynamics in sharply stratified ambient fluids, Phys. Rev. Fluids 1, 050503 (2016).

[3] C. Cohen, M. Berhanu, J. Derr, and S. Courrech du Pont, Erosion patterns on dissolving and melting bodies, Phys. Rev. Fluids 1, 050508 (2016).

[4] P. Chantelot, M. Coux, L. Domino, B. Pype, C. Clanet, A. Eddi, and D. Quéré, Kicked drops, Phys. Rev. Fluids 3, 100503 (2018).

[5] A. Bouillant, T. Mouterde, P. Bourrianne, C. Clanet, and D. Quéré, Symmetry breaking in Leidenfrost flows, Phys. Rev. Fluids 3, 100502 (2018).

[6] N. B. Speirs, M. M. Mansoor, J. Belden, R. C. Hurd, Z. Pan, and T. T. Truscott, Fluted films, Phys. Rev. Fluids 3, 100504 (2018).

[7] A. Krizhevsky, I. Sutskever, and G. E. Hinton, Imagenet classification with deep convolutional neural networks, in Advances in Neural Information Processing Systems (Curran Associates, Inc., Red Hook, NY, 2012), pp. 1097-1105.

[8] L. A. Gatys, A. S. Ecker, and M. Bethge, A neural algorithm of artistic style, Nat. Commun. (2015).

[9] A. Athalye, Neural style, https://github.com/anishathalye/neural-style (2015), commit 4ef159c. 